\title{
Dynamics of bed use in accommodating emergency admissions: stochastic simulation model
}

\author{
Adrian Bagust, Michael Place, John W Posnett
}

\begin{abstract}
Objective To examine the daily bed requirements arising from the flow of emergency admissions to an acute hospital, to identify the implications of fluctuating and unpredictable demands for emergency admission for the management of hospital bed capacity, and to quantify the daily risk of insufficient capacity for patients requiring immediate admission.

Design Modelling of the dynamics of the hospital system, using a discrete-event stochastic simulation model, which reflects the relation between demand and available bed capacity.

Setting Hypothetical acute hospital in England. Subjects Simulated emergency admissions of all types except mental disorder.

Main outcome measures The risk of having no bed available for any patient requiring immediate admission; the daily risk that there is no bed available for at least one patient requiring immediate admission; the mean bed occupancy rate.

Results Risks are discernible when average bed occupancy rates exceed about $85 \%$, and an acute hospital can expect regular bed shortages and periodic bed crises if average bed occupancy rises to $90 \%$ or more.

Conclusions There are limits to the occupancy rates that can be achieved safely without considerable risk to patients and to the efficient delivery of emergency care. Spare bed capacity is therefore essential for the effective management of emergency admissions, and its cost should be borne by purchasers as an essential element of an acute hospital service.
\end{abstract}

\section{Introduction}

Hospital provision for accommodating increasing numbers of emergency admissions is a matter of considerable public and political concern and has been the subject of widespread debate. ${ }^{2}$ For several years hospital managers have been under pressure to reduce bed capacity and increase occupancy rates in the name of operational efficiency. More recently, public disquiet has arisen in cases where patients could not gain ready access to a local hospital or were subjected to extended delays while vacant beds were identified. An appreciation of the dynamics of the hospital system in its ability to respond to fluctuating demand is important in framing policy, determining appropriate levels of service provision, and establishing realistic performance monitoring criteria.

Systematic analysis of long term trends in emergency admissions has been most fully developed in Scotland by Kendrick et al, ${ }^{3}{ }^{4}$ but no comparable series has been published for England. A detailed analysis of hospital episode statistics for all diagnoses (excluding mental health) in England for the period 1989-90 to 1995-6, undertaken as part of the project reported here, showed a trend line for emergency admissions rising at $1.9 \%$ a year, of which only a quarter was attributable to changes in the size and composition of the population. ${ }^{5}$ Elective admissions over the same period declined at a rate of $1.9 \%$ a year, while day cases rose by $16.3 \%$ a year. Occupied bed days declined by $4.6 \%$ a year for emergencies and by $5.4 \%$ a year for elective admissions.

Thus the balance between emergency and elective admissions has shifted in six years from $56 \%: 44 \%$ to 61\%:39\%, with emergency patients now occupying $65 \%$ of the staffed beds available. Hospitals are thus faced with an increasing proportion of their inpatient work coming from the fluctuating and unpredictable demands of emergency admissions, and less coming from planned elective patients.

Analysis of hospital episode statistics shows that emergency admissions will continue to rise, probably at a similar rate in the future to that experienced in the past. Equally, the proportion of emergency admissions is also likely to increase, so that the proportion of bed capacity required for emergency work will continue to grow.

Lane et al have examined the impact of hour by hour demand on an accident and emergency department, with particular emphasis on long waiting times for admission, ${ }^{6}$ but there is also a need to study the implications of the random nature of demand for emergency admission on a daily basis as it affects use of the bed stock. We are not aware of any recent published research with this emphasis.

Day to day fluctuations in emergency demand affect the quality of care and hospital efficiency, and an understanding of these effects is required to help in planning services and choosing operational interventions to alleviate problems and avoid crises.

\section{Methods}

Discrete-event stochastic simulation modelling is a well established management technique for investigating the behaviour of complex systems subject to random effects. ${ }^{7}$ Such a model was developed, using a Microsoft Excel 5 spreadsheet, to explore the relation between randomly fluctuating demand for patient admission and available inpatient bed capacity. The model generates new arrivals for each day of a 1000 day period as random variations around a long term trend line, using an empirically derived normal distribution adjusted for seasonal and day of week variations. These patients are then accommodated as long as the hospital has a vacant bed. The model is designed to allow complex interactions to be explored (for example, random shocks such as flu epidemics, or feedback between discharge and admission rates), but these features are not reported in this paper.

The model has been further extended to encompass elective admissions sharing a common bed pool
Paper $\mathrm{p} 158$
York Health
Economics
Consortium,
University of York,
Heslington, York
YO10 5DD
Adrian Bagust,
deputy director
Michael Place,
research fellow
John W Posnett,
director
Correspondence to:
Adrian Bagust
ab13@york.ac.uk
BMJ 1999;319:155-8 
Table 1 Baseline values in model of randomly fluctuating demand for patient admission and available inpatient bed capacity

\begin{tabular}{lc} 
Variable & Value \\
\hline Bed capacity: & 200 \\
\hline No of beds in emergency bed pool & 90 \\
\hline Initial bed occupancy (\%) & 5 \\
\hline Average beds temporarily unavailable (\%) & 24.7 \\
\hline Emergency admissions: & 7.5 \\
\hline Initial mean emergency admission rate (per day) & 3.5 \\
\hline Maximum seasonal variation in demand (\%) & \\
\hline Annual growth rate (\%) & 1.061 \\
\hline Mean day of week rate multipliers: & 1.064 \\
\hline Monday & 1.110 \\
\hline Tuesday & 1.048 \\
\hline Wednesday & 1.044 \\
\hline Thursday & 0.887 \\
\hline Friday & 0.783 \\
\hline Saturday & 0.44 \\
\hline Sunday & \\
\hline SD of random fluctuations & 14 \\
\hline Length of stay: & 12.75 \\
\hline Mean discharge rate (\%) (equivalent to length of stay of 7.14 days) & 0.5 day \\
\hline Percentage of admissions with zero length of stay & \\
\hline Notional bed days for zero length of stay & \\
\hline
\end{tabular}

(including some designated elective beds), and an additional performance measure has been introduced to indicate the proportion of elective admissions cancelled each day. However, the effect of this change proved limited-some emergencies are admitted at the expense of cancelled electives but with no increase in the occupancy rates for the emergency bed pool. The simplicity of the emergencies-only model is preferred here as it serves to focus attention on the primary problem of rising emergency admissions for acute hospitals.

The model was calibrated to a notional baseline position by using data obtained from detailed analysis of admissions records and length of stay distributions at two NHS trusts (one city centre teaching hospital and one non-teaching hospital in a semirural post-industrial town) and was adjusted to represent a situation where the hospital is just beginning to experience significant operational risks in relation to accommodating emergency arrivals. The baseline values are shown in table 1 .

Table 2 Factors varied in experiments using model of randomly fluctuating demand for patient admission and available inpatient bed capacity

\begin{tabular}{llc}
$\begin{array}{l}\text { Experiment } \\
\text { No }\end{array}$ & Factor varied & Range \\
\hline 1 & Initial emergency admissions rate as a proportion of total bed capacity & $5-15 \%$ \\
\hline 2 & Long term growth rate of emergency admissions & $0-6 \%$ \\
\hline 3 & Proportion of emergency patients with zero length of stay & $0-20 \%$ \\
\hline 5 & Notional stay ascribed to emergency patients classified as zero length of stay & $0-0.8$ days \\
\hline 6 & Daily discharge rate for emergency patients & $10-20 \%$ \\
\hline 7 & (Equivalent length of stay) & $(5-10$ days) \\
\hline 8 & Initial bed occupancy & $60-100 \%$ \\
\hline 9 & $\begin{array}{l}\text { Seasonal variation in emergency admissions as a proportion of mean } \\
\text { admission rate }\end{array}$ & $0-20 \%$ \\
\hline 10 & Standard deviation of the random variation in emergency admissions & $10-50 \%$ \\
\hline 11 & Magnitude of day of week variations in emergency admission rates (multiple \\
of baseline values) & $0.0-2.0$ \\
\hline & Number of beds temporarily unavailable (proportion of bed pool) & $0-20 \%$ \\
\hline
\end{tabular}
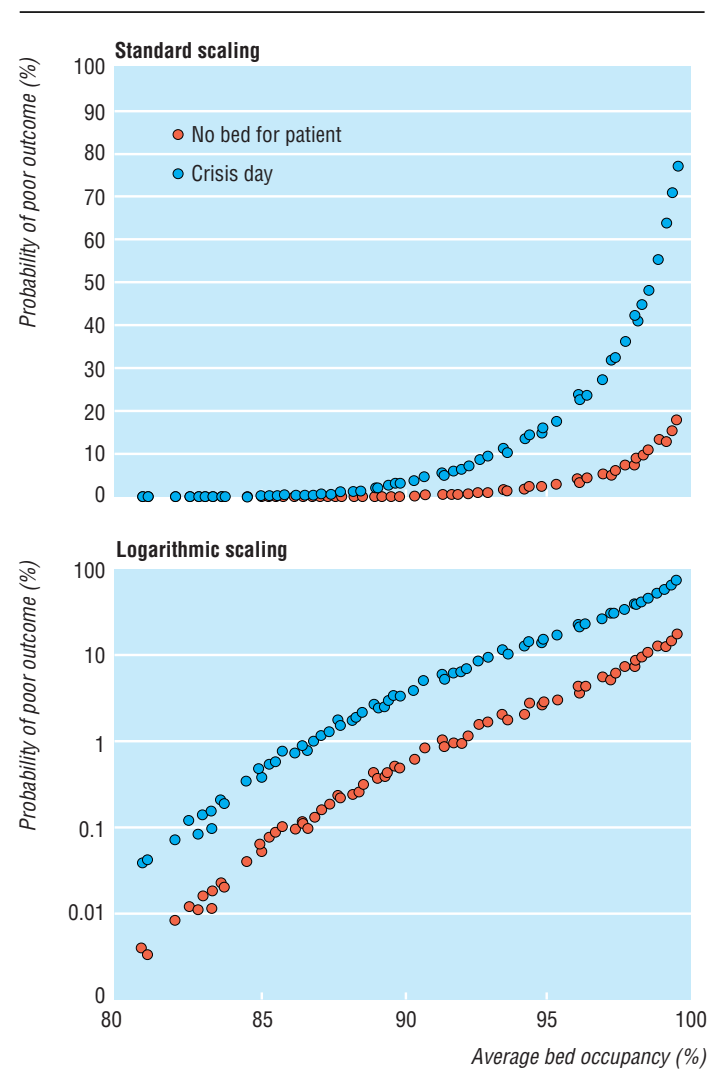

Relation of performance risks to average bed occupancy

A wide range of statistics can be derived from the model; the key output measures of system performance are the proportion of new arrivals for emergency admission who cannot be accommodated owing to lack of available beds; the proportion of days in a year when at least one patient requiring immediate admission cannot be accommodated (termed a crisis day); and the bed occupancy rates achieved. The level of risk to both patients and hospital is a function of the rate at which new emergency patients arrive at the hospital, the rate at which emergency patients are subsequently discharged, and the daily available bed capacity.

It was determined by experiment that a stable result was achieved when 100 repetitions of the model were carried out with different random numbers and the results aggregated. Eleven separate experiments were undertaken, in each of which a different factor was varied within a range of realistic values (table 2).

\section{Results}

Experiments 1-5 and experiment 10 were equivalent in terms of system dynamics; the figure shows the combined results. The level of risk for a hospital bed shortage is about four times that for an individual patient. Risks are minimal so long as the mean bed occupancy remains below about $85 \%$. Above this level the risks become substantial (at 85\%, a hospital can expect to be short of beds for admissions on four days in a year), and above a mean bed occupancy of $90 \%$ the system is regularly subject to bed crises.

Experiment 6 indicated that from very different initial bed occupancy positions all simulations con- 
verge to a relatively stable band within 14-16 days Thus a hospital that experiences a day on which no further admissions can be accommodated can expect to recover its long term demand-capacity balance in a period approximately equal to twice the mean length of stay. This implies that even a relatively low risk of failure can disrupt the operation of the hospital for a considerable time: at $85 \%$ mean occupancy, a hospital that runs out of beds for four days in a year may be disrupted for up to eight weeks in total.

The results of experiments 7-9 show that risk increases as variability rises, seasonality being the most influential factor. Varying the size of the bed pool over a wide range (experiment 11) affects risk only for capacities below about 150 beds, and even then produces only modest increases in risk.

\section{Discussion}

Management scientists have long been aware of the implications of queuing theory in systems with fixed or limited capacity. Thus our principal finding of a rapid increase in risk to patients and to the system at high occupancy levels is not unexpected.

Any model can capture only a limited range of the many contributory factors in real life, and models include various simplifying assumptions (such as the admission and discharge rate functions). Moreover we have intentionally reported only univariate experiments to show the relative importance of individual factors in the performance of the system. In reality these would all be operating together interactively, and additional complex organisational and psychological behaviours are likely to come into play as a hospital approaches maximum capacity. None the less, the results show how such responses may affect the system: if consultants seek to protect beds against mounting demand by delaying discharges, this will probably hasten the onset of a crisis, whereas action to discharge patients early may allow a crisis to be temporarily delayed.

\section{Demand and capacity}

The relation between demand, capacity, and the risk of failure is reflected in the average occupancy rate. At rates above $85 \%$ risks become discernible, and above $90 \%$ the hospital system is subject to regular bed crises. The average occupancy rate in acute hospitals in England was $78.9 \%$ in $1996-7,{ }^{8}$ and this is probably typical of the whole NHS. At a projected rate of growth in emergency bed days of $2.5 \%$ a year, this suggests that the NHS as a whole may be operating at $85 \%$ occupancy currently, and could exceed $90 \%$ by $2002-3$.

However, little is known about the causes of the exogenous upward trend in the demand for emergency admission. Most suggested interventions have not been evaluated, and evidence on their relative effectiveness is scant. Indeed, some forms of intervention (educational programmes, telephone advice, admissions wards, etc) may actually increase admissions. $^{9-11}$

\section{Effect of interventions}

It is important to distinguish between one-off interventions with only a short term impact and interventions that alter underlying trends. For example, the introduc- tion of a rapid response team will not have a long term effect unless the capacity of the team is increased annually in line with the increase in demand.

Possible interventions to avoid or alleviate the effects of rising emergency admissions can be grouped into four categories: avoiding admissions (fewer patients presenting to the hospital), alternatives to admission (more non-hospital options available when patients present), better management of existing resources, and facilitating early discharge. The potential benefits of each can be assessed in the light of our findings from the model.

Policies designed to reduce the rate of growth in the demand for emergency admissions and those designed to provide alternatives to admission in the acute sector offer greatest long term benefit. Reducing the rate of growth of admissions (experiment 2) has the same effect as an equivalent annual increase in capacity (experiments 1 and 11). By combining the modelling results with future projections based on our analysis of hospital episode statistics, ${ }^{5}$ we estimate that a reduction in the annual rate of growth of admissions of $1 \%$ has the effect of postponing by one year the time at which average occupancy in the acute sector reaches $85 \%$ and by three years the time at which average occupancy reaches $90 \%$.

Management interventions designed to improve the efficiency with which current resources are used have the effect of increasing capacity (experiment 1). Such policies should be part of good management practice in all trusts, but most if not all of these interventions can be expected to lead to a one-off impact with little effect on the long term trend.

Facilitating earlier discharge has the effect of increasing capacity by reducing the average length of stay (experiment 5). However, the potential for further sustained reductions in average length of stay is likely to be finite and the long term potential of this type of policy is therefore limited.

It might be expected that where local hospitals cooperate in pooling capacity the situation could be eased. This may be true if different hospitals in the same area experience different pressures on the same day. However, if two units draw from the same catchment area, there may be little net benefit as they will only be reorganising the same capacity within a combined bed pool (experiment 11). It should also be recognised that where several hospitals are all operating close to maximum capacity, a crisis in one hospital can quickly be transmitted through the whole of the local system-the domino effect.

\section{Difficulties in evaluation}

The stochastic nature of emergency admissions has an important implication for attempts to evaluate management interventions. The performance of an acute hospital can vary considerably from one year to another solely as a result of the large fluctuations in demand, swamping the effects of policy or operational initiatives. Hence the meaningful evaluation of any measures taken to improve the performance of a single acute hospital in relation to emergency admissions is not possible unless effects are monitored over a long period (typically 5-10 years) or across a very large sample of hospitals; year on year comparisons for one hospital are meaningless. The difficulty of carrying out 


\section{Key messages}

- Acute hospitals which operate at bed occupancy levels of $90 \%$ or more face regular bed crises, with the associated risks to patients

- Management interventions should focus on measures with long term benefits to counteract the growth trend in demand for admission

- Many initiatives have only a short term effect; they briefly delay the worst effects but do not address the growing mismatch between supply and demand

- Evaluating management interventions year on year at a single hospital is futile-any effects are swamped by random variation

such evaluations is an important justification for a modelling approach.

Emergency admissions are, by their nature, stochastic and difficult to predict. Our model shows that spare capacity is essential if an emergency admissions service is to operate efficiently and at a level of risk acceptable to patients. Emergency admission crises are not generally created by poor management. With insufficient spare bed capacity even the best run hospital is at risk. It must be recognised by the NHS that maintaining some unoccupied staffed beds is not wasteful, but is a cost which must be incurred if a quality service is to be sustained.

The work reported here formed part of a larger project undertaken in conjunction with the Department of Health Sciences and Clinical Evaluation at the University of York, Coventry Business School, and Plymouth University. The invaluable assistance and support of Andrea Roalfe, senior analyst at the NHS Executive (West Midlands), is gratefully acknowledged.

Contributors: $\mathrm{AB}$ directed the design of the model, carried out the experiments, synthesised the results, and drafted and edited the paper; MP developed and tested the model structure, carried out data analysis and model calibration, and participated in writing and editing the paper; JWP initiated the project, contributed to conceptual discussions, analysed the policy implications, and participated in writing and editing the paper. All authors are guarantors for the paper.

Funding: NHS Executive, West Midlands.

Competing interests: None declared.

1 Capewell S. The continuing rise in emergency admissions. BMJ 1996;312:991-2

2 Blatchford O, Capewell S. Emergency medical admissions: taking stock and planning for winter. BMJ 1997;315:1322-3

3 Kendrick S. The pattern of increase in emergency hospital admissions in Scotland. Health Bull 1996;54:169-83.

4 Kendrick S, Frame S, Povey C. Beds occupied by emergency patients: long term trends in patterns of short term fluctuations in Scotland. Health Bulletin 1997;55:167-75.

5 Crossen-White H, Moss P, Morris D, Di Blasi Z, Lambert M, Russell I, et al. The rise in emergency admissions. Report to the West Midlands NHS Executive, 1998. (Available from York Health Economics Consortium, University of York.)

6 Lane DC, Monefeldt C, Rosenhead JV. Looking in the wrong place for healthcare improvements: a system dynamics study of an accident and emergency department. London: London School of Economics, 1998. (LSE working paper series LSEOR 98.23.)

7 Pidd M. Computer simulation in management science, 4th edition. Chichester: Wiley, 1998.

8 Government Statistical Service. NHS hospital activity statistics: England 1986 to 1996-97. London: Department of Health, 1997. (Department of Health Statistical Bulletin 1997/20.)

9 Eppler E, Eisenberg MS, Schaeffer S, Meischke H, Larson MP. 911 and emergency department use for chest pain: results of a media campaign. Ann Emerg Med 1994;24:202-8.

10 Shaughnessy PW, Tynan EA. The use of swing beds in rural hospitals. Inquiry 1985;22:303-15.

11 MacLaren RE, Ghoorahoo HI, Kirby NG. Use of an accident and emergency department observation ward in the management of head injury. BrJ Surg 1993;80:215-7.

(Accepted 4 May 1999)

\title{
The rise in emergency admissions-crisis or artefact? Temporal analysis of health services data
}

\author{
Kieran Morgan, David Prothero, Stephen Frankel
}

Paper $\mathrm{p} 155$

Avon Health Authority, Bristol BS2 8EE

Kieran Morgan, director of public health

David Prothero, senior statistician Department of Social Medicine, University of Bristol, Bristol BS8 2PR

Stephen Frankel, professor of epidemiology and public health medicine Correspondence to: Stephen Frankel stephen.frankel@ bris.ac.uk

BMJ 1999;319:158-9
It is a common view that emergency admissions are increasing at up to $5 \%$ per year in the United Kingdom, ${ }^{1}$ and that this unsustainable rise "threatens the future of the NHS." The perceived rise in emergency admissions is invoked to explain those recurrent and well publicised crises that in turn support the view that there is a fundamental mismatch between demand and supply in health care, ${ }^{3}$ as the reported trend is held to represent a real and substantial increase in demand for hospital care.

\section{Subjects, methods, and results}

The data presented here reflect all emergency admissions in all medical and surgical specialties from 1989-90 to 1997-8 in an urban and rural population of 850000 served by Avon Health Authority. Three trends are described: numbers of people receiving hospital treatment each year; numbers of admissions each year, where readmissions are additional events (admissions are here provider spell admissions, where transfer between hospitals within a trust remains a single admission); and episodes, or more correctly, finished consultant episodes, which constitute a continuous period under an individual consultant's care.

Episodes of emergency treatment have risen $4.4 \%$ a year over the period, but the number of admissions has increased by only $2.0 \%$ a year (figure). The number of people receiving emergency treatment has increased only slightly, at an annual rate of $1.4 \%$, of which an increase of some $0.6 \%$ could be expected from the increase in the numbers of older people in the population during this period. A rise of some $0.8 \%$ in emergency admission therefore remains unexplained.

Though the ratio of episodes to admissions increased from 1.06 to 1.32 over the period, the overall readmission rate, as summarised by the ratio of admissions to individuals, has remained relatively constant, rising from 1.17 to 1.22. During the period the average length of stay per emergency admission fell from 10.2 